\title{
GROUP DECOMPOSITION BY DOUBLE COSET MATRICES
}

\section{J. SUTHERLAND FRAME}

1. Introduction. We are concerned in this paper with a study of some important properties of the matrices $V$ of the commuting ring of a finite intransitive group of permutation matrices $R$ whose transitive constituents we denote by $R^{s}, R^{t}, \ldots$. In particular we shall find an explicit method for obtaining the degrees and characters of the common irreducible components of two permutation representations $R^{s}$ and $R^{t}$ of a group $G$.

Our interest in this subject was first aroused in connection with the study of molecular structure, in which theoretical chemists have made use of the theory of groups. The potential energy of the molecule is approximated by a quadratic form whose matrix $V$ must be commutative with all the matrices of the symmetry group of the molecule. Its characteristic roots are closely related to the molecular spectrum. This suggests the general problem: "What matrices are permutable with a given intransitive symmetry group?"

Let $G$ then be any finite group of order $g$, represented by an intransitive permutation group $R$,

$$
R=R^{1}+\cdots+R^{s}+R^{t}+\cdots,
$$

which is the direct sum of transitive constituents $R^{s}, R^{t}, \ldots$ The rectangular matrices $V^{t s}$ which intertwine $R^{t}$ and $R^{s}$,

$$
R^{t} V^{t s}=V^{t s} R^{s}
$$

are submatrices of the matrices $V$ which commute with $R$. One basis for these matrices $V^{t s}$ is supplied by the $\mu^{s t}$ double coset matrices $V_{\alpha}^{t s}$ described in $\S 2$. An example is given in $\$ 3$ to illustrate the theory at this point.

After a change of basis by suitable unitary transformations $U^{s}, U^{t}, \cdots$ obtained in $\S 4$, the permutation groups $R^{s}, R^{t}$ are rewritten as completely reduced groups,

$$
R_{U}^{8}=\left(U^{8}\right)^{-1} R^{8} U^{8}=\cdot \sum_{j} F_{j} \times I_{j}^{8},
$$

which are the direct sums of $\left(f_{j} \mu_{j}^{s}\right)$-dimensional square blocks placed consecutively along the diagonal. In each block $R_{U}^{s}$ is the direct

Presented to the Society, December 28, 1946; received by the editors August 29, 1947. 
product of an irreducible representation $F_{j}$ of degree $f_{j}$ by a unit matrix $I_{j}^{s}$ of degree $\mu_{j}^{s}$. Under this same change of basis the double coset matrices $V_{\alpha}^{t s}$ of the set $V^{t s}$ are transformed into rectangular basis matrices $\Phi_{\alpha}^{t s}$ of the transformed linear set $\Phi^{t s}$,

$$
\Phi_{\alpha}^{t_{s}}=\left(U^{t}\right)^{-1} V_{\alpha}^{t_{s}} U^{s}=\cdot \sum_{j} I_{j}^{0} \times \Phi_{\alpha j}^{t_{s}},
$$

where $I_{j}^{0}$ is the unit matrix of degree $f_{j}=\mu_{j}^{0}$ (corresponding to the identity subgroup $H^{0}$ ) and where $\Phi_{\alpha j}^{t s}$ are rectangular matrices whose coefficients $\phi_{\alpha j, \rho \sigma}^{t s}\left(\rho=1 \cdots \mu_{j}^{t} ; \sigma=1 \cdots \mu_{j}^{s}\right)$ we shall call double coset coefficients. Theorem 1 in $\$ 3$ gives an explicit formula for the double coset coefficients in terms of the entries of the irreducible components $F_{j}$. Later we try in reverse to obtain information about the $F_{j}$ by first computing the double coset coefficients.

The double coset coefficients define a transformation from the basis $\Phi_{\alpha}^{t s}$ of $\Phi^{t s}$ to a new basis $E_{\eta}^{t s}$ described in $\S 5$. We use the single subscript $\eta$ to replace the subscripts $j, \rho, \sigma$ arranged in dictionary order.

$$
\Phi_{\alpha}^{t_{s}}=\sum_{\eta} \rho_{\alpha \eta}^{t_{s}} E_{\eta}^{t_{s}} ; \quad \rho_{\alpha \eta}^{t_{s}}=\phi_{\alpha j, \rho \sigma}^{t_{s}}
$$

We shall prove in Theorem 2 of $\$ 5$ that the double coset coefficients $\rho_{\alpha \eta}^{t s}$ satisfy a set of orthogonality relations, which include as a special case the well known orthogonality relations among the coefficients of the irreducible representations $F_{j}$ of $G$.

Similarly in $\S 6$ the double coset traces $\tau_{\alpha j}^{t}$ and their associated factors $\psi_{\alpha \jmath}^{k}$,

$$
\begin{aligned}
\tau_{\alpha j}^{t} & =\sum_{\sigma} \phi_{\alpha j, \sigma \sigma}^{t t}, \\
\psi_{\alpha j}^{t} & =\tau_{\alpha j}^{t} \tau_{\alpha 1}^{t} \tau_{1 j}^{t} ; \quad \psi_{\alpha 1}^{t}=1,
\end{aligned}
$$

are shown to satisfy orthogonality relations including the well known relations among the group characters. The double coset traces are proved in Theorem 3 to be algebraic integers, and certain group characters are obtained as linear combinations of the $\psi_{\alpha j}^{t}$.

In $\$ 7$ we assume that some properties of the permutation groups $R^{s}$ and $R^{t}$ and the related subgroups $H^{s}$ and $H^{t}$ are known, but not the irreducible representations $F_{j}$. The problem is to find the degrees and characters of those $F_{j}$ which are common components of $R^{s}$ and $R^{t}$. The answer is given in terms of the double coset traces and the numbers $k_{\alpha \lambda}^{s t}$ which define the number of elements of $G$ common to a double coset $H_{\alpha}^{s t}$ and a class of conjugates $C_{\lambda}$. For subgroups of rela- 
tively small index in $G$, for which $\mu^{s t}$ is small, the double coset coefficients can easily be determined by factoring a determinant of order $\mu^{s t}$ using our principal Theorem 4 . The characters of the $F_{j}$ can then be obtained by a direct and unambiguous computation.

2. Double coset matrices and the commuting ring. Let $\gamma_{1}=1$, $\gamma_{2}, \cdots, \gamma_{0}$ be the elements of a finite group $G$, which is represented as the intransitive group of permutation matrices (1.1). Let $H^{s}$ denote the subgroup consisting of the $h^{s}$ elements of $G$ which leave invariant the first symbol permuted by the matrices of $R^{s}$, and let $\theta_{i}^{s}\left(i=1,2, \cdots, n^{s}\right)$ be the right coset element in the group ring of $G$ which is the sum of the elements of the right $\operatorname{coset} H^{s} \gamma_{i}$. In particular, $\theta_{1}^{s}$ represents the sum of the elements in the subgroup $H^{s}$, and $\theta_{1}^{s} / h^{s}$ is an idempotent of the group ring. If $h_{\alpha}^{s t}$ denotes the order of the intersection of $\gamma_{\alpha}^{-1} H^{s} \gamma_{\alpha}$ and $H^{t}$, and $n_{\alpha}^{s t}$ the index of this subgroup in $G$, then we define the double coset element $\theta_{\alpha}^{\text {st }}$ of the group ring by 1

$$
\theta_{\alpha}^{s t}=\theta_{\alpha}^{s} \theta_{1}^{t} / h_{\alpha}^{s t}=n_{\alpha}^{s t} \theta_{\alpha}^{s} \theta_{1}^{t} / g=\theta_{1}^{s} \gamma_{\alpha} \theta_{1}^{t} / h_{\alpha}^{s t} .
$$

This element $\theta_{\alpha}^{s t}$ is the sum of $h^{t} / h_{\alpha}^{s t}$ distinct right coset elements $\theta_{i}^{s}$. Each element of $G$ occurs as a summand in exactly one of the $\theta_{\alpha}^{s t}$, and occurs there with coefficient 1 . We form a column vector $\theta^{t}$ of $n^{t}$ rows having $\theta_{i}^{l}$ in the $i$ th row, and a column vector $\theta$ of $n^{1}+n^{2}+\cdots$ rows which is the direct sum of $\theta^{1}, \theta^{2}, \ldots$. The transitive groups of matrices $R^{t}$ and their intransitive direct sum $R$ can then be defined:

$$
\begin{aligned}
& \theta^{t} \gamma_{i}=R^{t}\left(\gamma_{i}\right) \theta^{t}, \\
& \theta \gamma_{i}=\left(\begin{array}{c}
\theta^{1} \\
\theta^{2} \\
: \\
:
\end{array}\right) \gamma_{i}=\left(\begin{array}{cccc}
R^{1}\left(\gamma_{i}\right) & 0 & \cdots & 0 \\
0 & R^{2}\left(\gamma_{i}\right) & \cdots & 0 \\
\vdots & \vdots & \ldots & . \\
: & \vdots & \cdots & :
\end{array}\right)\left(\begin{array}{c}
\theta^{1} \\
\theta^{2} \\
\vdots \\
:
\end{array}\right)=R\left(\gamma_{i}\right) \theta .
\end{aligned}
$$

By applying the associative law and (2.3) to the product $\theta \gamma_{i} \gamma_{j}$ we verify that $R$ is homomorphic with $G$, since

$$
R\left(\gamma_{i}\right) R\left(\gamma_{j}\right)=R\left(\gamma_{i} \gamma_{j}\right) .
$$

The double coset matrix $V_{\alpha}^{t s}$ is defined by the formula

$$
\theta_{\alpha}^{s t} \theta^{t}=h^{t} V_{\alpha}^{t_{s}} \theta^{s} .
$$

${ }^{1}$ A. Speiser, Die Theorie der Gruppen von endlicher Ordnung. The notion of double cosets is explained on p. 63. 
The entries of $V_{\alpha}^{t s}$ consist of $h^{t} / h_{\alpha}^{s t} 1$ 's in each of its $n^{t}$ rows, and $h^{s} / h_{\alpha}^{s t}$ 1 's in each of its $n^{s}$ columns, all other entries being 0's. There are $n_{\alpha}^{s t}$ 1 's altogether in a particular $V_{\alpha}^{t s}$. Since the sum over $\alpha$ of $\theta_{\alpha}^{s t}$ is the same as the sum of all elements of $G$, the sum over $\alpha$ of all $V_{\alpha}^{t s}$ is a rectangular matrix of 1 's.

The fact that $V_{\alpha}^{t s}$ intertwines the matrices $R^{t}\left(\gamma_{i}\right)$ and $R^{s}\left(\gamma_{i}\right)$ is seen by combining (2.2) and (2.5). The scalar $\theta_{\alpha}^{s t}$ is permutable with the matrix $R^{t}\left(\gamma_{i}\right)$ and we have

$$
\begin{aligned}
R^{t}\left(\gamma_{i}\right) V_{\alpha}^{t s} h^{t} \theta^{s} & =R^{t}\left(\gamma_{i}\right) \theta_{\alpha}^{s t} \theta^{t}=\theta_{\alpha}^{s t} R^{t}\left(\gamma_{i}\right) \theta^{t}=\theta_{\alpha}^{s t} \theta^{t} \gamma_{i}=h^{t} V_{\alpha}^{t s} \theta^{s} \gamma_{i} \\
& =V_{\alpha}^{t s} R^{s}\left(\gamma_{i}\right) h^{t} \theta^{s} .
\end{aligned}
$$

Comparing coefficients of $h^{t} \theta^{s}$, we have an equation similar to (1.2):

$$
R^{t}\left(\gamma_{i}\right) V_{\alpha}^{t s}=V_{\alpha}^{t s} R^{s}\left(\gamma_{i}\right)
$$

If $V$ is any matrix in the commuting ring of $R$ we have

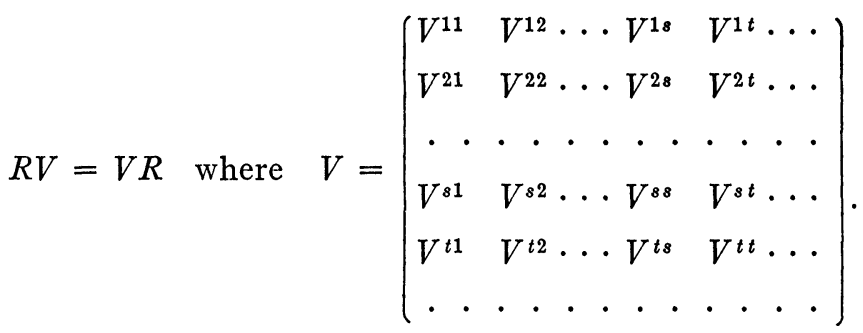

It has been shown in an earlier paper ${ }^{2}$ that the submatrix $V^{t s}$ of (2.8) which satisfies (1.2) can be expressed as a linear combination of the $\mu^{s t}$ double coset matrices $V_{\alpha}^{t s}$,

$$
V^{t_{s}}=\sum_{\alpha} c_{\alpha}^{t_{s}} V_{\alpha}^{t_{s}}
$$

Furthermore the transpose of the matrix $V_{\alpha}^{t s}$ is the matrix $V_{\alpha}^{s t}$ which corresponds to the inverse double coset $H_{\alpha^{\prime}}^{t s}$,

$$
\left(V_{\alpha}^{t s}\right)^{\prime}=V_{\alpha^{\prime}}^{s t} .
$$

The identity element of $G$ will occur in the product $H_{\beta^{\prime}}^{t s} H_{\alpha}^{s t}$ only if $\beta=\alpha$. Hence the trace ( $\operatorname{tr})$ of the product $V_{\alpha}^{t s} V_{\beta^{\prime}}^{s t}$ is zero unless $\alpha=\beta$. It is then equal to $n_{\alpha}^{s t}$, the number of 1 's in the matrix $V_{\alpha}^{t s}$. Thus

$$
\operatorname{tr}\left(V_{\alpha}^{t s} V_{\beta^{\prime}}^{s t}\right)=\delta_{\alpha \beta} n_{\alpha}^{s t} .
$$

2 J. S. Frame, Double coset matrices and group characters, Bull. Amer. Math. Soc. vol. 49 (1943) pp. 81-92. 
3. An example of double cosets. In order to fix ideas we illustrate the theory with an example in which $G$ is the symmetric group of degree 4 and order 24 whose elements $\gamma_{i}$ we denote by writing them as permutations on 4 digits. Let $\theta_{1}^{s}=I+(13)$ and $\theta_{1}^{t}=I+(13)+(24)$ $+(13)(24)$, so that $H^{s}$ and $H^{t}$ are subgroups of order 2 and 4 respectively. Then the right cosets and double cosets are as follows.

(3.1) Right cosets

$$
\begin{array}{lll}
\theta_{1}^{s}=I+(13) & \theta_{12}^{s}=(13)(24)+(24) & \theta_{1}^{t}=\theta_{1}^{s}+\theta_{12}^{s} \\
\theta_{2}^{s}=(143)+(34) & \theta_{11}^{s}=(234)+(1423) & \theta_{2}^{t}=\theta_{2}^{s}+\theta_{11}^{s} \\
\theta_{3}^{s}=(134)+(14) & \theta_{10}^{s}=(142)+(1342) & \theta_{3}^{t}=\theta_{3}^{s}+\theta_{10}^{s} \\
\theta_{4}^{s}=(123)+(23) & \theta_{9}^{s}=(243)+(1243) & \theta_{4}^{t}=\theta_{4}^{s}+\theta_{9}^{s} \\
\theta_{5}^{s}=(132)+(12) & \theta_{8}^{s}=(124)+(1324) & \theta_{5}^{t}=\theta_{5}^{s}+\theta_{8}^{s} \\
\theta_{6}^{s}=(12)(34)+(1432) & \theta_{7}^{s}=(14)(23)+(1234) & \theta_{6}^{t}=\theta_{6}^{s}+\theta_{7}^{s}
\end{array}
$$

(3.2) Double cosets

$$
\begin{array}{lll}
\theta_{1}^{s s}=\theta_{1}^{s} & \theta_{1}^{s t}=\theta_{1}^{s}+\theta_{12}^{s} & \theta_{1}^{t s}=\theta_{1}^{t} \\
\theta_{2}^{s s}=\theta_{2}^{s}+\theta_{3}^{s} & \theta_{2}^{s t}=\theta_{2}^{s}+\theta_{3}^{s}+\theta_{8}^{s}+\theta_{9}^{s} & \theta_{2}^{t s}=\theta_{2}^{t}+\theta_{3}^{t} \\
\theta_{3}^{s 8}=\theta_{4}^{s}+\theta_{5}^{s} & \theta_{3}^{s t}=\theta_{4}^{s}+\theta_{5}^{s}+\theta_{10}^{s}+\theta_{11}^{s} & \theta_{3}^{t s}=\theta_{4}^{t}+\theta_{5}^{t} \\
\theta_{4}^{s s}=\theta_{6}^{s}+\theta_{7}^{s} & \theta_{4}^{s t}=\theta_{6}^{s}+\theta_{7}^{s} & \theta_{4}^{t s}=\theta_{6}^{t} \\
\theta_{5}^{s s}=\theta_{8}^{s}+\theta_{9}^{s} & & \theta_{1}^{t t}=\theta_{1}^{t} \\
\theta_{6}^{s s}=\theta_{10}^{s}+\theta_{11}^{s} & & \theta_{2}^{t t}=\theta_{2}^{t}+\theta_{3}^{t}+\theta_{4}^{t}+\theta_{5}^{t} \\
\theta_{7}^{s s}=\theta_{12}^{s} & & \theta_{3}^{t t}=\theta_{6}^{t}
\end{array}
$$

Typical permutation matrices $R^{s}(\gamma)$ and $R^{t}(\gamma)$ for $\gamma=(143)$ are

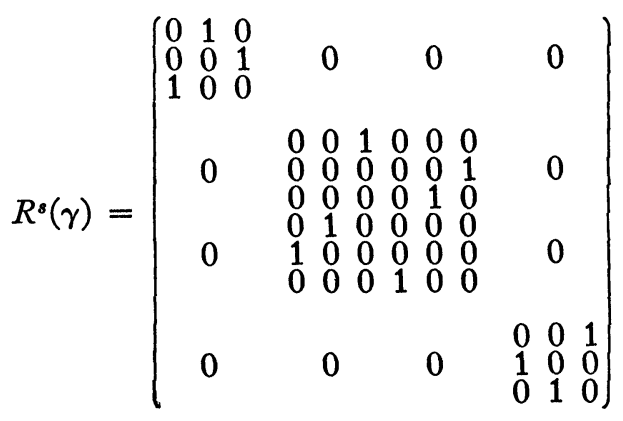




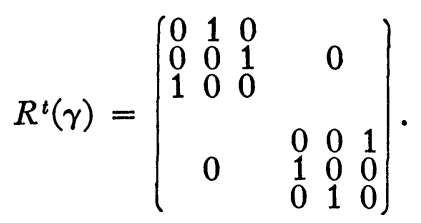

In writing the general double coset matrix $V$ it will be easier to print and to read if we replace the arbitrary parameters $c_{1}^{s s} \cdots c_{7}^{s s}$ by letters $A B C D E F G$, replace $c_{1}^{s t} \cdots c_{4}^{s t}$ by $X Y Z W$, replace $c_{1}^{t s} \cdots c_{4}^{t s}$ by $x y z w$, and replace $c_{1}^{t t}, c_{2}^{t t}, c_{3}^{t t}$, by $a b c$ respectively. Then the matrix $V$ becomes

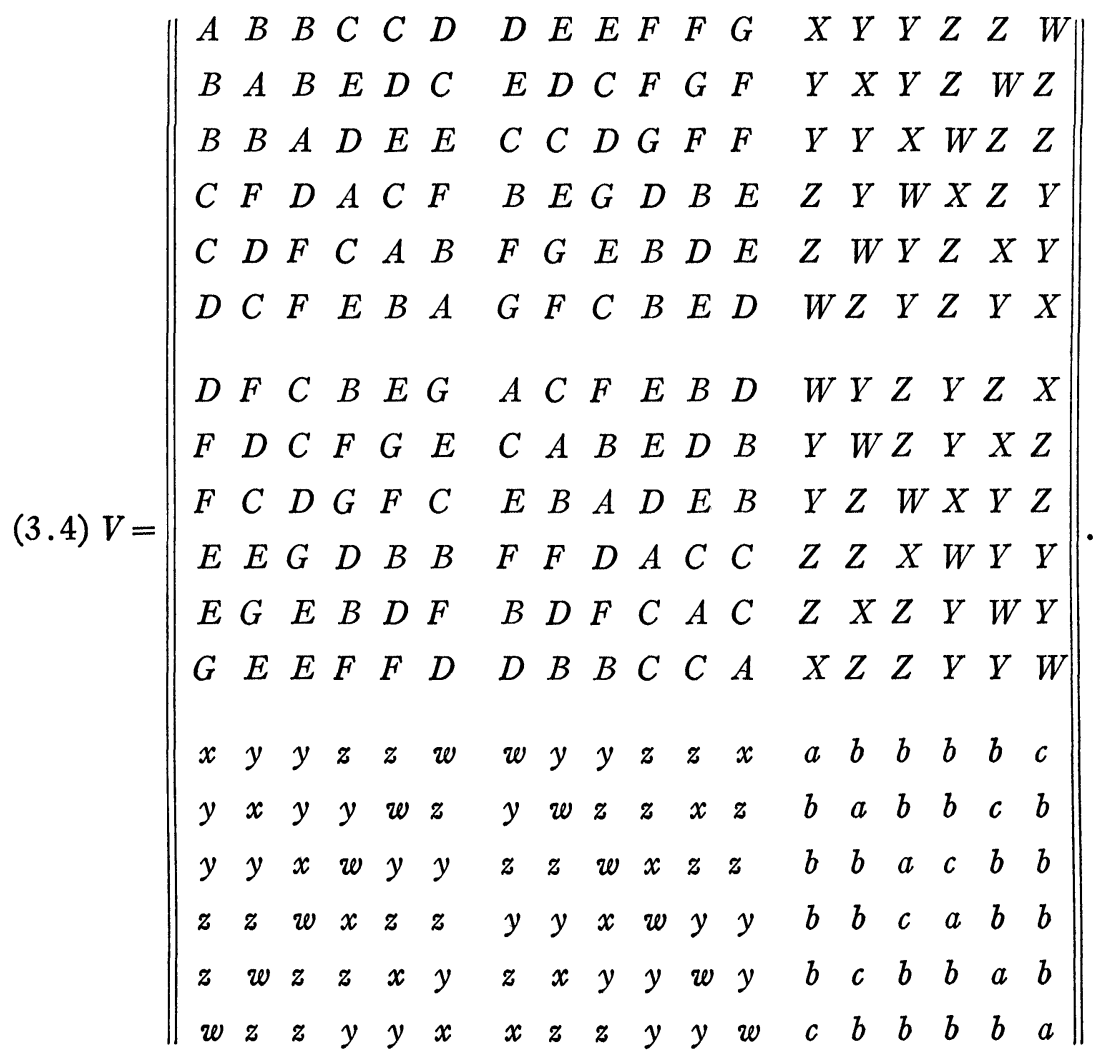

A particular double coset matrix is obtained by setting one of the letters in (3.4) equal to 1 and all the rest 0.

4. The transformation to irreducible components. A finite group $G$ with $k$ classes of conjugates $C_{\lambda}$ has exactly $k$ non-equivalent sets of irreducible representations as a group of unitary transformations of 
a vector space over a field $K$ of characteristic 0 which includes the $g$ th roots of unity. Let $F_{j}$ of degree $f_{j}$ be a particular unitary representation in the $j$ th set, and denote by $f_{j, \rho \sigma}\left(\gamma_{i}\right)$ the entry in the $\rho$ th row and $\sigma$ th column of the matrix representing $\gamma_{i}$ in $F_{j}$. We order the $g=\sum f_{i}^{2}$ sets of three indices $j, \rho, \sigma$ in dictionary order and assign to them the single index $\eta$. Given $\eta$ we shall write $\eta^{*}$ to denote the subscript $j$ of the corresponding $F_{j}$. The complete set of coefficients in all the selected $F_{j}$ for each $\gamma_{i}$ may then be written in a $g \times g$ matrix $Z$ with entries

$$
z_{i \eta}=f_{i, \rho \sigma}\left(\gamma_{i}\right) .
$$

From $Z$ we derive a unitary matrix $T$ with entries

$$
t_{i \eta}=z_{i \eta}\left(f_{\eta^{*}} / g\right)^{1 / 2}
$$

which completely reduces both the right and left regular representations of $G .^{3}$

From $T$ in turn we shall derive a decompossable unitary matrix

$$
U_{*}^{*}=\left(X^{s}\right)^{-1} T Q^{*}=\left(\begin{array}{ll}
U^{*} & 0 \\
0 & *
\end{array}\right)
$$

which is adapted to the subgroup $H^{s}$ and contains in its upper left corner an $n^{s} \times n^{s}$ matrix $U^{s}$ which reduces $R^{s}$ according to (1.3). This we do as follows.

Denoting by $\theta^{0}$ a column vector whose entries are the elements $\gamma_{1} \cdots \gamma_{\theta}$ of $G$, and by $\gamma$ an arbitrary element of the group ring of $G$, the regular representations $R^{0}$ and $L$ are given by

$$
\theta^{0} \gamma=R^{0}(\gamma) \theta^{0} ; \quad \gamma \theta^{0}=L(\gamma) \theta^{0} .
$$

We define a new basis vector $\epsilon$ such that $\theta^{0}=T \epsilon$. Then

$$
\epsilon \gamma=T^{-1} R^{0}(\gamma) T \epsilon ; \quad \gamma \epsilon=T^{-1} L(\gamma) T \epsilon .
$$

We further define $I_{j}$ to be the idempotent matrix which vanishes except for $f_{j}^{2} 1$ 's as diagonal entries where $\eta^{*}=j$. Then

$$
\begin{aligned}
I_{j} T^{-1} R^{0}(\gamma) T I_{j} & =F_{j}(\gamma) \cdot \times I_{j}^{0}, \\
I_{j} T^{-1} L(\gamma) T I_{j} & =I_{i}^{0} \times F_{j}^{\prime}(\gamma)
\end{aligned}
$$

where $I_{j}^{0}$ is a unit matrix of degree $f_{j}$ and where $\times$ denotes the left direct product in which the submatrices consist of the first matrix

${ }^{3} \mathrm{~J}$. S. Frame, On the reduction of the conjugating representation of a finite group, Bull. Amer. Math. Soc. vol. 53 (1947) p. 589. 
multiplied by an entry from the second, ${ }^{4}$ and the prime denotes the transposed matrix. Now $F_{j}\left(\theta_{1}^{s} / h^{s}\right)$ is an idempotent matrix of rank $\mu_{j}^{s}$, so we may find a suitable unitary matrix $Q_{j}^{s}$ which will bring it to the form $I_{j}^{s}=\operatorname{diag}(1,1, \cdots, 1,0, \cdots, 0)$. The matrices

$$
F_{j}^{8}(\gamma)=\left(Q_{j}^{8}\right)^{-1} F_{j}(\gamma) Q_{j}^{8}
$$

will be said to be adapted to the subgroup $H^{8}$. The direct sum of the products $I_{j}^{0} \times \times\left(\bar{Q}_{j}^{s}\right)$ is now multiplied by a permutation matrix to give a product $Q^{s}$ which assembles the $n^{s}=\mu_{j}^{s} f_{j}$ nonvanishing entries of the idempotents for $H^{s}$ so that the matrix

$$
L^{s}=\left(Q^{8}\right)^{-1} T^{-1} L\left(\theta_{1}^{8} / h^{\circ}\right) T Q^{8}=\left(\begin{array}{ll}
I^{8} & 0 \\
0 & 0
\end{array}\right)
$$

is a diagonal idempotent having 1 's in its first $n^{s}$ diagonal entries, and 0 's elsewhere.

Furthermore $T Q^{s}$ transforms $R^{0}(\gamma)$ into a matrix having the submatrix $R^{s}$ in the upper left corner. It is permutable with $L^{s}$ and hence decomposable.

Having adapted the irreducible representations $F_{j}$ to the subgroup $H^{s}$ we next adapt the basis vector $\theta^{0}$ to $H^{s}$. Let $P^{s}$ be a permutation matrix such that in the vector $\left(P^{s}\right)^{-1} \theta^{0}$ the $q$ th element of the $\nu$ th right coset of $H^{s}$ in $G$ occupies the $i=(q-1) n^{8}+\nu$ row. Set

$$
X^{s}=P^{s} Y^{s}, \quad Y^{s}=I^{s} \bullet \times T^{s}
$$

where $Y^{s}$ is the left direct product of a unit matrix of degree $n^{s}$ with the unitary matrix $T^{s}$ of degree $h^{s}$ which completely reduces the regular representation of $H^{s}$. In its first $n^{s}$ columns the matrix $\left(h^{s}\right)^{1 / 2} Y^{s}$ contains $I^{s}$ repeated $h^{s}$ times. The conjugate transposed matrix is $\left(h^{s}\right)^{1 / 2}\left(Y^{s}\right)^{-1}$. This collects the coset elements of $\left(P^{s}\right)^{-1} \theta^{0}$ together, so that the new basis vector

$$
\zeta^{s}=\left(h^{s}\right)^{1 / 2}\left(X^{s}\right)^{-1} \theta^{0}
$$

contains in its first $n^{s}$ rows the subvector $\theta^{s}$ previously defined, and contains in its other rows ring elements which are annihilated on left multiplication by $\theta_{1}^{s}$ or any $\theta_{\alpha}^{t s}$. From (2.5) and (4.10) we obtain

$$
\begin{aligned}
\left(\begin{array}{ll}
V_{\alpha}^{t s} & 0 \\
0 & 0
\end{array}\right) \zeta^{s} & =\left(h^{t}\right)^{-1} \theta_{\alpha}^{s t} \zeta^{t}=\left(h^{t}\right)^{-1 / 2}\left(X^{t}\right)^{-1} \theta_{\alpha}^{s t} \theta^{0} \\
& =\left(h^{t}\right)^{-1 / 2}\left(X^{t}\right)^{-1} L\left(\theta_{\alpha}^{s t}\right) \theta^{0} .
\end{aligned}
$$

\footnotetext{
4 C. C. MacDuffee, Theory of matrices, Chelsea Press, 1947.
} 
Expressing $\theta^{0}$ in terms of $\zeta^{s}$ by (4.10) and comparing coefficients we have

$$
\left(\begin{array}{ll}
V_{\alpha}^{t_{s}} & 0 \\
0 & 0
\end{array}\right)=\left(X^{t}\right)^{-1} L\left(\theta_{\alpha}^{s t}\right) X^{s}\left(h^{s} h^{t}\right)^{-1 / 2} .
$$

Using the $X^{s}$ of (4.9) we define the unitary matrix $U_{*}^{s}=\left(X^{s}\right)^{-1} T Q^{s}$ as in (4.3), and then multiply each member of (4.12) on the left by $\left(U_{*}^{t}\right)^{-1}$ and on the right by $U_{*}^{s}$. Thus we have

$$
\left(U_{*}^{t}\right)^{-1}\left(\begin{array}{ll}
V_{\alpha}^{t_{s}} & 0 \\
0 & 0
\end{array}\right) U_{*}^{s}=\left(Q^{t}\right)^{-1} T^{-1} L\left(\theta_{\alpha}^{s t}\right) T Q^{s}\left(h^{s} h^{t}\right)^{-1 / 2} .
$$

Since the right member of (4.13) belongs to the left ideal generated by the idempotent $L^{s}$ of (4.8) and to the right ideal generated by $L^{t}$, it follows that the unitary matrices $U_{*}^{s}$ and $U_{*}^{t}$ are decomposable as indicated in (4.3). It can be shown by a straightforward computation that the submatrix $U^{s}$ reduces $R^{s}$ as in (1.3). Also by (4.13) we may express the transformed double coset matrices $\Phi_{\alpha}^{\text {ts }}$ of (1.4) in terms of the coefficients in $F_{j}$ by the equation

$$
\left(\begin{array}{ll}
\Phi_{\alpha}^{t s} & 0 \\
0 & 0
\end{array}\right)=\left(Q^{t}\right)^{-1} T^{-1} L\left(\theta_{\alpha}^{s t}\right) T Q^{s}\left(h^{s} h^{t}\right)^{-1 / 2} .
$$

Since $T$ is a unitary matrix which completely reduces $L$ as shown in (4.6) it follows that both members of (4.14) actually are reduced, as was stated in the second part of (1.4). Consequently $\Phi_{\alpha \jmath}^{t s}$ is a $\mu_{j}^{t} \times \mu_{f}^{s}-$ dimensional submatrix of the matrix

$$
\left(Q_{j}^{t \prime}\right) F_{j}^{\prime}\left(\theta_{\alpha}^{s t} /\left(h^{8} h^{t}\right)^{1 / 2}\right) \bar{Q}_{j}^{s} .
$$

Taking the transpose of (4.15) and using (4.7) we have

$$
\left(\begin{array}{cc}
\Phi_{\alpha i}^{t_{s}} & 0 \\
0 & 0
\end{array}\right)^{\prime}=\left(\begin{array}{cc}
I_{j}^{s} & 0 \\
0 & 0
\end{array}\right)\left(Q_{i}^{s}\right)^{-1} F_{i}\left(\gamma_{\alpha} \rho_{\alpha 1}^{s t}\right) Q_{j}^{t}\left(\begin{array}{cc}
I_{j}^{t} & 0 \\
0 & 0
\end{array}\right)
$$

where

$$
\rho_{\alpha 1}^{s t}=\left(h^{8} h^{t}\right)^{1 / 2 t} h_{\alpha}^{s t} .
$$

TheOREM 1. The transpose of the $\mu_{j}^{i} \times \mu_{j}^{s}$-dimensional submatrix $\Phi_{\alpha j}^{t s}$ of the double coset matrix $\Phi_{\alpha}^{\text {ts }}$ may be expressed in terms of the coefficients of the irreducible representation $F_{j}$ of $G$ by equation (4.16).

The factor $\rho_{\alpha 1}^{t s}$ in (4.16) is a mean proportion between the number of 
right cosets of $H^{s}$ and the number of left cosets of $H^{t}$ in the double coset $H_{\alpha}^{s t}$. The group element $\gamma_{\alpha}$ is any element in this double coset.

5. Orthogonality relations. Having transformed $R$ and $V$ by the unitary matrix $U$ which is the direct sum of the matrices $U^{s}$ defined in (4.3) we now investigate certain algebraic properties of the double coset matrices $\Phi_{\alpha}^{t s}$. We express the latter in terms of the new basis matrices $E_{\eta}^{t s}$ defined by (1.5).

The matrix $E_{\eta}^{s t}$ consists entirely of 0 's except for $f_{j} 1$ 's forming a unit matrix $I_{j}^{0}$ in the rows corresponding to the $\rho$ th occurrence of $F_{j}$ as a component of $R_{U}^{t}$, and columns correspond to the $\sigma$ th occurrence of $F_{j}$ as a component of $R_{U}^{s}$. The number of distinct matrices $E_{\eta}^{t s}$ for fixed $t s$ is $\sum_{j} \mu_{j}^{t} \mu_{j}^{s}$. This is equal to the number $\mu^{s t}$ of double cosets $V_{\alpha}^{t s}$, which has been called the transitive factor of the two subgroups $H^{s}$ and $H^{t} .{ }^{5}$

Solving for $E_{\zeta}^{t s}$ in terms of $\Phi_{\alpha}^{t s}$ we write

$$
E_{\zeta}^{t_{s}}=\sum_{\beta} \sigma_{\zeta \beta}^{t_{8}} \Phi_{\beta}^{t_{s}}
$$

defining $\left(\sigma_{\zeta \beta}^{t s}\right)$ to be the inverse matrix of $\left(\rho_{\alpha \eta}^{t s}\right)$. Denoting by $E_{\zeta^{\prime}}^{s t}$ the transpose of the real matrix $E_{\zeta}^{t s}$, and taking the transposed conjugates in (5.1) we have

$$
E_{\zeta^{\prime}}^{s t}=\sum_{\beta} \bar{\sigma}_{\zeta \beta}^{t s} \Phi_{\beta^{\prime}}^{s t}
$$

where $\Phi_{\beta^{\prime}}^{s t}$ and $\Phi_{\beta}^{t s}$ correspond to inverse double cosets.

The trace of the product $E_{\eta}^{t s} E_{\xi^{\prime}}^{s t}$ is $f_{\eta^{*}} \delta_{\eta \xi}$, whereas the trace of the product $\Phi_{\alpha}^{t s} E_{\zeta^{\prime}}^{s t}$ may be expressed in two ways, by using first (1.5) and then (5.2) and (2.11).

$$
\begin{aligned}
& \operatorname{tr}\left(\Phi_{\alpha}^{t_{s}} E_{\zeta^{\prime}}^{s t}\right)=\sum_{\eta} \rho_{\alpha \eta}^{t_{s}} \operatorname{tr}\left(E_{\eta}^{t_{s}} E_{\zeta^{\prime}}^{s t}\right)=\rho_{\alpha \zeta}^{t_{s}} f_{\zeta^{*}} \\
& =\sum_{\beta} \bar{\sigma}_{\zeta \beta}^{t s} \operatorname{tr}\left(\Phi_{\alpha}^{t s} \Phi_{\beta^{\prime}}^{s t}\right) \\
& =\sum_{\beta} \bar{\sigma}_{\zeta \beta}^{t_{s}} \operatorname{tr}\left(V_{\alpha}^{t_{s}} V_{\beta^{\prime}}^{s t}\right)=\bar{\sigma}_{\zeta \alpha}^{t_{s}} n_{\alpha}^{t_{s}} \text {. }
\end{aligned}
$$

Hence

$$
\rho_{\alpha \zeta}^{t_{s}} f_{\zeta^{*}}=\bar{\sigma}_{\zeta \alpha}^{t_{s}} n_{\alpha}^{t_{s}} .
$$

Two sets of orthogonality relations follow from (5.4).

${ }^{5}$ D. E. Littlewood, The theory of group characters, Oxford, 1940, p. 165. L. E. Dickson, Algebras, and their arithmetics, Chicago, 1923, 


$$
\begin{aligned}
& \sum_{\alpha} h_{\alpha}^{s t} \rho_{\alpha \eta}^{t_{0}} \bar{\rho}_{\alpha \zeta}^{t_{s}}=\left(g / f_{\zeta^{*}}\right) \delta_{\eta \zeta} \text {. } \\
& \sum_{\zeta} f_{\zeta^{*} \rho_{\alpha \zeta_{i} \beta \zeta}}^{t_{s}-t_{s}}=\left(g / h_{\alpha}^{s t}\right) \delta_{\alpha \beta}=n_{\alpha}^{s t} \delta_{\alpha \beta} .
\end{aligned}
$$

Equations (5.5) and (5.6) both reduce to the orthogonality relations among coefficients of $F_{j}$ if $H^{s}=H^{t}=1$. They may be expressed by the statement:

THEOREM 2. The $\mu^{\text {st }}$-dimensional square matrix, whose entries are the modified double coset coefficients $\rho_{\alpha \zeta}^{t s}\left(f_{5^{*}} / n_{\alpha}^{t s}\right)^{1 / 2}$, is a unitary matrix.

6. The double coset traces. Although the double coset coefficients may depend on the particular choice of matrices $Q_{j}^{s}$ which are used to adapt the representation $F_{j}$ to the subgroup $H^{s}$, the traces $\tau_{\alpha j}^{b}$ of the double coset submatrices $\Phi_{\alpha j}^{t}$ of (1.4) and (4.16) are invariants of the group and are uniquely determined. They were defined in (1.6).

Theorem 3. The double coset traces are algebraic integers.

PRoof. The determinant $\left|\sum p_{\alpha}^{t} V_{\alpha}^{t t}\right|$ is a form of degree $n^{t}$ in the parameters $p_{\alpha}^{t}$ with integral coefficients. After transforming the basis by $U^{t}$, the new matrix $\Phi_{\alpha}^{t t}$ breaks up into the direct sum of submatrices $\Phi_{\alpha j}^{z t}$ of degree $\mu_{j}^{t}$ and multiplicity $f_{j}$. Hence

$$
\left|\sum_{\alpha} p_{\alpha}^{t} V_{\alpha}^{t t}\right|=\left|\sum_{\alpha} p_{\alpha}^{t} \Phi_{\alpha}^{t t}\right|=\prod_{i}\left|\sum_{\alpha} p_{\alpha}^{t} \Phi_{\alpha i}^{t t}\right|^{f_{i}} .
$$

The absolutely irreducible factors $\left|\sum p_{\alpha}^{t} \Phi_{\alpha j}^{t}\right|$ of the rational integral form $\left|\sum p_{\alpha}^{z} V_{\alpha}^{u}\right|$ must have coefficients which are algebraic integers in the extension field $K$ in which the latter form factors. Expanding a given irreducible factor in powers of $p_{1}^{t}$, and noting that $\Phi_{1 j}^{t}$ is a unit matrix, we have

$$
\left|\sum p_{\alpha}^{t} \Phi_{\alpha j}^{t t}\right|=\left(p_{1}^{t}\right)^{\mu_{j}}+\left(p_{1}^{t}\right)^{\mu_{j-1}} \sum_{\alpha \neq 1} p_{\alpha}^{t} \tau_{\alpha j}^{t}+\cdots .
$$

Hence $\tau_{\alpha j}^{t}$ is an algebraic integer for $\alpha \neq 1$. The trace of the $\mu_{j}^{t}$-dimensional unit matrix is the integer $\tau_{1 j}^{t}=\mu_{j}^{z}$. Q. E. D.

Orthogonality relations for the double coset traces can be derived from (5.5) by setting $s=t$ and summing over values of $\eta$ and $\zeta$ corresponding to diagonal elements in $\Phi_{\alpha \mu t}^{t s}$. We obtain

$$
\sum_{\alpha} h_{\alpha}^{t t} \tau_{\alpha j}^{t} \bar{\tau}_{\alpha k}^{t}=\left(g \mu_{j}^{t} / f_{j}\right) \delta_{j k} \text {. }
$$

These become the usual relations for group characters if $H^{t}$ is the 
identity subgroup. Since

$$
\tau_{\tau_{i j}}^{t}=\mu_{j}^{t}, \text { and } \tau_{\alpha 1}^{t}=h^{t} / h_{\alpha}^{t t}=n_{\alpha}^{t t} / n^{t},
$$

it is convenient whenever $\mu_{j}^{t} \neq 0$ to introduce the associated trace factors $\psi_{\alpha\}}^{2}$,

$$
\psi_{\alpha j}^{t}=\tau_{\alpha j}^{t} / \tau_{\alpha 1}^{t} \tau_{1 j}^{t}
$$$$
\text { where } \psi_{\alpha 1}^{t}=1
$$

We may then rewrite (6.3) in the form

$$
\sum_{\alpha} \tau_{\alpha j}^{t} \bar{\psi}_{\alpha k}^{t}=\left(n^{t} / f_{j}\right) \delta_{j k}, \quad \text { if } \mu_{j}^{t} \neq 0 .
$$

This equation will be useful later in finding the degrees $f_{j}$.

We define the following quantities

$$
\chi_{\lambda}^{\prime}=\text { trace of } F_{j}(\gamma) \text { for } \gamma \text { in the class } C_{\lambda} \text {. }
$$

$$
g_{\lambda}=\text { number of elements of } G \text { in } C_{\lambda} \text {. }
$$

$g_{\alpha \lambda}^{t}=$ number of elements of $C_{\lambda}$ in the right $\operatorname{coset} H^{t} \gamma_{\alpha}$.

$k_{\alpha \lambda}^{t}=g_{\alpha \lambda}^{t} \tau_{\alpha 1}^{t}$, number of elements of $C_{\lambda}$ in the double coset $H_{\alpha}^{t}$.

Using the expression (4.15) for $s=t$ and thus computing the trace $\tau_{\alpha j}^{t}$ of the $\mu_{j}^{z}$-dimensional matrix $\Phi_{\alpha j}^{u}$ we find a relation between the double coset traces and group characters.

$$
\tau_{\alpha i}^{t}=\sum_{\lambda}\left(k_{\alpha \lambda}^{t} / h^{t}\right) \chi_{\lambda}^{j}=\tau_{\alpha 1}^{t} \sum_{\lambda}\left(g_{\alpha \lambda}^{t} / h^{t}\right) \chi_{\lambda}^{i} .
$$

Solving for $\bar{\psi}_{\alpha k}$ from (6.8) and (6.5), we substitute in (6.6) and multiply by $h^{t}$.

$$
\sum_{\alpha, \mu} g_{\alpha \mu}^{t} \tau_{\alpha i}^{t} \bar{\chi}_{\mu}^{k}=\tau_{1 k}^{t}\left(g / f_{j}\right) \delta_{j k}
$$

Multiplying by $\chi_{\lambda}^{k}$ in (6.9) and employing the orthogonality of group characters, we have after summing over $k$ and solving for $\chi_{\lambda}^{1}$,

$$
\chi_{\lambda}^{j}=\left(f_{j} / g_{\lambda}\right) \sum_{\alpha} k_{\alpha \lambda}^{t} \psi_{\alpha j}^{t} .
$$

This equation expresses the group characters in terms of the associated trace factors.

7. Determination of the degrees and characters of the irreducible representations. In a paper entitled Double coset matrices and group characters (abbreviated DCM) ${ }^{2}$ the author defined the non-negative 
integral multiplication constants $c_{\alpha \beta \eta}^{r s t}$ for the double cosets by

$$
H_{\alpha}^{r s} H_{\beta^{\prime}}^{s t} / h^{s}=\sum_{\eta} c_{\alpha \beta \eta}^{r s t} H_{\eta}^{r t}
$$

and denoted by $K^{s t}$ the matrix whose elements in row $\alpha$ and column $\beta$ are the linear functions $\sum c_{\alpha \beta \eta}^{s t} b_{\eta}^{t}$ of the parameters $b_{\eta}^{t}$ :

$$
K^{s t}=\left(\sum_{\eta} c_{\alpha \beta \eta}^{t s t} b_{\eta}^{t}\right) .
$$

It was stated erroneously on p. 82 of DCM that the determinant of $n^{t} K^{s t}$ factors into irreducible factors which are linear in the parameters $b_{\eta}^{t}$. The irreducible factors are indeed linear in the new parameters $a_{\lambda}$ when we replace $b_{\eta}^{t}$ by

$$
b_{\eta}^{t}=\sum_{\lambda} k_{\eta \lambda}^{t} a_{\lambda}
$$

and this was the statement made on p. 90 of DCM in the principal theorem of the paper. But for multiplicities $\mu_{j}^{t}>1$ the corresponding irreducible factors are of degree $\mu_{j}^{t}$ and reduce to linear factors only when the $b_{\eta}^{t}$ are suitably restricted as in (7.3).

The correct general statement of the factoring of the determinant is given by the following theorem.

THEOREM 4. The determinant of the matrix of linear combinations $\sum_{\alpha} c_{\rho \sigma \alpha}^{s t} n_{\alpha}^{t z} p_{\alpha}^{t}$ of double coset structure constants, in which $p_{\alpha}^{i}$ are arbitrary parameters and $\rho, \sigma$ represent row and column indices, can be factored into the irreducible double coset factors $\left|\sum_{\alpha} p_{\alpha}^{i} \Phi_{\alpha \jmath}^{u}\right|$ of degree $\mu_{j}^{t}$ occurring with multiplicities $\mu_{j}^{s}$. The numerical coefficient of the $\mu^{s t}$ power of $p_{1}^{t}$ is the product of the $n_{\alpha}^{t}$, and this is equal to the product of $f_{j}$ to the power $\mu_{j}^{s} \mu_{j}^{t}$ multiplied by an integer which is divisible by $n^{s} n^{t}$. This integer is the squared absolute value of the determinant $\left|\rho_{\alpha \eta}^{s t}\right|$.

Furthermore, after making the substitution

$$
p_{\alpha}^{t}=\sum_{\lambda} g_{\alpha \lambda}^{t} a_{\lambda}
$$

the factors of degree $\mu_{j}^{t}$ each reduce to a power of a factor which is linear in the group characters; thus

$$
\left|\sum p_{\alpha}^{t} \Phi_{\alpha j}^{t t}\right|=\left(\sum_{\lambda} a_{\lambda} g_{\lambda} \chi_{\lambda}^{j}\right)^{\mu_{j}^{t}}
$$

Stated in formulas we have 


$$
\begin{aligned}
\mid \sum_{\alpha} c_{\rho \sigma \alpha}^{t s t} n_{\alpha}^{t t} p_{\alpha}^{t} & =n^{s} n^{t} A^{s t} \prod_{j} f_{j}^{\mu_{j \mu j}^{t}}\left|\sum_{\alpha} p_{\alpha}^{t} \Phi_{\alpha j}^{t t}\right|^{\mu_{j}^{s}}, \\
\mid \sum_{\alpha, \lambda} c_{\rho \sigma \alpha}^{t s t} n_{\alpha}^{t t} g_{\alpha \lambda}^{t} a_{\lambda} & =n^{s} n^{t} A^{s t} \prod_{j}\left(f_{i} \sum_{\lambda} a_{\lambda} g_{\lambda} \chi_{\lambda}^{j}\right)^{\mu_{j}^{s} \mu_{j}^{t}}
\end{aligned}
$$

where $A^{\text {st }}$ is the integer

$$
A^{s t}=\left|\rho_{\alpha \eta}^{s t}\right||| \bar{\rho}_{\alpha \eta}^{s t} \mid / n^{s} n^{t} .
$$

Proof of Theorem 4. Following the line of argument of pp. 87 and 89 of DCM we evaluate the trace (tr) of a matrix in two ways. Taking $p_{\alpha}^{t}$ as arbitrary parameters we have

$$
\begin{aligned}
\mathrm{X}_{\rho \sigma}^{s t}(p)=\operatorname{tr}\left(V_{\rho^{\prime}}^{t s} V_{\sigma}^{s t} \sum_{\alpha} p_{\alpha}^{t} V_{\alpha}^{t t}\right) & =\operatorname{tr}\left(\sum_{\beta, \alpha} c_{\rho \sigma \beta}^{t s t} V_{\beta^{\prime}}^{t t} V_{\alpha}^{t t} p_{\alpha}^{t}\right) \\
& =\sum_{\alpha} c_{\rho \sigma \alpha}^{t s t} n_{\alpha}^{t t} p_{\alpha}^{t} .
\end{aligned}
$$

Transforming by $U$ and applying (1.5) we have

$$
\begin{aligned}
\mathrm{X}_{\rho \sigma}^{s t}(p) & =\operatorname{tr}\left(\Phi_{\rho^{\prime}}^{t_{s}} \Phi_{\sigma}^{s t} \sum_{\alpha} p_{\alpha}^{t} \Phi_{\alpha}^{t t}\right) \\
& =\sum_{\zeta, \eta} \bar{\rho}_{\rho \zeta}^{s t} \rho_{\sigma \eta}^{s t} \operatorname{tr}\left(E_{\zeta^{\prime}}^{t_{s}} E_{\eta}^{s t} \sum_{\alpha} p_{\alpha}^{t} \Phi_{\alpha}^{t t}\right)
\end{aligned}
$$

where as usual the subscripts $\eta$ and $\zeta$ each stand for three indices $j, \lambda \mu$, and so on. Now the trace in (7.10) vanishes for all pairs of values $\zeta, \eta$ except those corresponding to the same $j$ and $\lambda$. If $\zeta$ is $j, \lambda \nu$ and $\eta$ is $j, \lambda \mu$ we obtain a nonvanishing trace only from the component of $\Phi_{\alpha j}^{t t}$ in the $\mu$ th set of $f_{j}$ rows and the $\nu$ th set of $f_{j}$ columns. The trace is then equal to $f_{j} \sum_{\alpha} p_{\alpha}^{t} \phi_{\alpha j, \mu \nu}^{t}$. For each $j$ there are $\mu_{j}^{s}$ values of $\lambda$. Hence the matrix $\mathbf{X}_{\rho \sigma}^{s t}(p)$ may be written as the product of three matrices in which the first and last factors are $\left(\rho_{\sigma \eta}^{s t}\right)$ and its transposed conjugate, and the second matrix is the direct sum of the matrices $f_{j} \sum p_{\alpha}^{t} \Phi_{\alpha j}^{t t}$ each occurring as a summand with multiplicity $\mu_{j}^{s}$. Comparing determinants of (7.9) and (7.10) and using the formula (7.8) which was proved in DCM, we obtain the furst stated result.

If the $p_{\alpha}^{\imath}$ are expressed as linear combinations of $a_{\lambda}$ by (7.4), however, then the matrices $\sum p_{\alpha}^{t} \Phi_{\alpha j}^{t}$ become diagonal matrices and their determinants factor into linear factors (7.5) as proved in DCM. The comparison of numerical coefficients is immediate, ${ }^{6}$ since $p_{1}^{t}$ occurs

${ }^{6} \mathrm{~J}$. S. Frame, The double cosets of a finite group, Bull. Amer. Math. Soc. vol. 47 (1941) p. 459, Theorem B. 
only along the principal diagonals of $X_{\rho \sigma}^{s t}(p)$ and of $\sum p_{\alpha}^{t} \Phi_{\alpha \jmath}^{t}$. Q.E.D.

To apply Theorem 4 to the determination of group characters let us suppose that $H^{s}$ and $H^{t}$ are two large subgroups of $G$ such that the number of common irreducible components $F_{j}$ of $R^{s}$ and $R^{t}$ is small, and $\mu^{s t}$ is small, possibly not more than 5 or 6 , preferably smaller yet. Then if the structure of the group $G$ is known it is not difficult to obtain the constants $c_{\rho \sigma \alpha}^{t s t}$ from the multiplication of double cosets. Factoring the determinant as in (7.6) we obtain the double coset traces $\tau_{\alpha j}^{t}$ as coefficients of $\left(p_{1}^{l}\right)^{m-1} p_{\alpha}^{t}$ in an irreducible factor of degree $m=\mu_{j}^{t}$ in which $\left(p_{1}^{t}\right)^{m}$ has coefficient 1 . The associated factors $\psi_{\alpha j}^{t}$ are found from (6.5), the degrees $f_{j}$ from (6.6), and finally the characters themselves from (6.10).

In simple cases where a subgroup $H^{t}$ has only three or four double cosets we may even compute the double coset traces directly without using the multiplication constants $c_{\rho \sigma \alpha}^{s t}$. We must then have $\mu_{j}^{t}=\tau_{1,}^{t}=1$, $\sum_{j} \tau_{\alpha j}^{l}=0$ for $\alpha \neq 1$, and we know the $\tau_{\alpha 1}^{t}$ from (6.4). We substitute from (6.5) into (6.6) and try to find algebraic integers $\tau_{\alpha j}^{l}$ for which $\tau_{\alpha j}^{t}$ and $\bar{\psi}_{\alpha k}^{t}$ are orthogonal $(j \neq k)$ and for which

$$
f_{i}=\frac{n^{t}}{\sum_{\alpha} \tau_{\alpha j}^{t} \bar{\tau}_{\alpha j} / \tau_{\alpha 1}^{t}}
$$

is an integer. If no two irreducible components $F_{j}$ occurring in $R_{U}^{s}$ are algebraically conjugate, the $\tau_{\alpha j}^{l}$ will be rational integers such that $\sum_{\alpha} \tau_{\alpha j}^{t}=0$ for $j \neq 1$.

In the example on pp. $190-192$ in DCM we had $\mu^{s t}=2, n^{t}=45$. Expressed in the terms of parameters $p_{\alpha}$ the two linear factors of the determinants (7.6) are then equal to $\left(p_{1}+12 p_{2}+32 p_{3}\right)$ and $\left(p_{1}+3 p_{2}-4 p_{3}\right)$. Hence the matrices $\left(\tau_{\alpha \jmath}^{t}\right)$ and $\left(\psi_{\alpha \jmath}^{t}\right)$ are

$$
\left(\tau_{\alpha j}^{t}\right)=\left\|\begin{array}{rr}
1 & 1 \\
12 & 3 \\
32 & -4
\end{array}\right\|, \quad\left(\psi_{\alpha j}^{t}\right)=\left\|\begin{array}{cc}
1 & 1 \\
1 & 1 / 4 \\
1 & -1 / 8
\end{array}\right\|
$$

and we find immediately $f_{1}=1, f_{2}=45 /(1+9 / 12+16 / 32)=20$, $\chi_{\lambda}^{(2)}=20\left(g_{1 \lambda}+3 g_{2 \lambda}-4 g_{3 \lambda}\right)$.

Similarly, considering the permutation group of the 27 lines on the cubic surface, we have just three double cosets, with $\tau_{\alpha 1}^{s}=1,10,16$. Hence $\mu^{8 s}=3$. Either $\tau_{23}$ or $\tau_{33}$ is non-negative. Suppose it is $\tau_{23}$. Setting $\tau_{23}=x$ and $\tau_{32}=y$ we must find non-negative integral solutions $(x, y)$ of the equation

(7.13) $1-y(x+1) / 10-x(y+1) / 16=0$, or $13 x y+5 x+8 y=80$. 
Hence $x y<6$, and $y(x+1) \equiv 0(\bmod 5)$. The solutions for $(x, y)$ are $(0,10),(16,0)$ and $(4,1)$. Only the last choice gives integral values for $f_{j}$ and we then have by (6.5) and (7.11),

$$
\begin{gathered}
\left(\tilde{\tau}_{\alpha j}^{\circ}\right)=\left\|\begin{array}{rrr}
1 & 1 & 1 \\
10 & -5 & 1 \\
16 & 4 & -2
\end{array}\right\|, \quad\left(\psi_{\alpha j}^{*}\right)=\left\|\begin{array}{ccc}
1 & 1 & 1 \\
1 & -1 / 2 & 1 / 10 \\
1 & 1 / 4 & -1 / 8
\end{array}\right\|, \\
f_{2}=\frac{27}{4.5}=6, \\
f_{3}=\frac{27}{1.35}=20 .
\end{gathered}
$$

The irreducible components have degrees $1,6,20$, and the characters may be found by applying (6.10).

Michigan State College

\section{EQUAL SUMS OF LIKE POWERS}

\section{E. M. WRIGHT}

Let $s \geqq 2$ and let $P(k, s)$ be the least value of $j$ such that the equations

$$
\sum_{i=1}^{j} a_{i 1}^{h}=\sum_{i=1}^{j} a_{i 2}^{h}=\cdots=\sum_{i=1}^{j} a_{i s}^{h} \quad(1 \leqq h \leqq k)
$$

have a nontrivial solution in integers, that is, a solution in which no set $\left\{a_{i u}\right\}$ is a permutation of another set $\left\{a_{i v}\right\}$. It was remarked by Bastien [1] ${ }^{1}$ that $P(k, 2) \geqq k+1$ and this is true $a$ fortiori for general $s$. The only upper bound for $P(k, s)$ for general $k$ and $s$ which I have found in the literature is due to Prouhet [5] who (in 1851) gave solutions of (1) with $j=s^{k}$, so that $P(k, s) \leqq s^{k}$. He allocates each of the numbers $0,1, \cdots, s^{k+1}-1$ to the set $\left\{a_{i u}\right\}$ if the sum of its digits in the scale of $s$ is congruent to $u(\bmod s)$. Recently Lehmer [4] took $m_{1}, \cdots, m_{k+1}$ any $k+1$ integers, let each of $b_{1}, \cdots, b_{k+1}$ run through 1947.

Presented to the Society, October 25, 1947; received by the editors September 23,

1 Numbers in brackets refer to the bibliography at the end of the paper. 